\title{
Estudo anatômico da válvula do seio coronário: válvula de Thebesius
}

\author{
Fábio B. JATENE*, Reinaldo de Brito COSTA SOBRINHO*, Silvia Regina Trovareli ROMERO*, Adib D. \\ JATENE*
}

RBCCV 44205-88

JATENE, F. B.; COSTA SOBRINHO, R. B.; ROMERO, S. R. T.; JATENE, A. D. - Estudo anatómico da válvula do seio coronário: válvula de Thebesius. Rev. Bras. Cir. Cardiovasc., 4(2): 163-167, 1989.

RESUMO: Este estudo tem por objetivo esclarecer eventuais problemas causados pela presença da válvula de Thebesius durante a cateterização do seio coronário, quer pela cardioplegia retrógrada, estudo eletrofisiológico, ou eletrofulguraçăo. Foram analisados 94 coraçōes normais, de óbitos nâo cardiacos. 0 átrio direito era incisado ao nível da veia cava superior, em direçăo à veia cava inferior, percorrendo o sulco terminal, possibilitando adequada visibilização do seio coronário. As válvulas, classificadas de acordo com suas variaçōes anatómicas, possibilitaram o seguinte resultado: ausente em $15(16 \%)$ casos; residual em $31(33 \%)$; parcial em $40(43 \%)$; trabeculada em sete $(7 \%)$ e dupla em um $(1 \%)$ caso. A cateterizaçăo do seio coronário não foi prejudicada quando este apresentava válvula ausente, residual ou dupla. Entretanto, em alguns dos casos onde havia válvula parcial (11 de 40 casos, $27 \%$ ) e válvula trabeculada (trés de sete casos, $43 \%$ ), esta cateterização foi mais trabalhosa e demorada. Estatisticamente, não houve correlação dos tipos de válvula com relação a sexo e raça, sendo inconclusiva com relação à idade.

DESCRITORES: válvula de Thebesius, anatomia.

\section{INTRODUÇĀO}

A válvula do seio coronário, ou válvula de Thebesius, é uma simples dobra semicurcular da membrana de revestimento do átrio, inserida na borda direita e inferior do orifício do seio coronário ${ }^{3}$.

Pela disposição anatômica, poderia ser considerada como um dispositivo anti-refluxo para o seio coronário. muito embora, na prática, isso não ocorra, sendo que autores como TESTUT \& LATARJET $^{7}$ a consideram mesmo como uma relíquia anatômica. Entretanto, nos dias atuais, vem sendo reconsiderada, devido ao uso da cardioplegia realizada através do seio coronário ${ }^{4}$. Como se sabe, estenoses coronárias e insuficiência aórtica interferem muito na efetividade da cardioplegia realizada por via anterógrada, levando, muitas vezes, a uma inadequada preservação da musculatura cardíaca du- rante $\mathrm{o}$ ato cirúrgico. A cardioplegia retrógada surge como opção para este tipo de problema, embora, quando realizada diretamente no átrio direito, provoque uma dilatação desta cavidade, bem como do ventrículo direito ${ }^{1}$. Esta problema pode ser minimizado pela infusão da solução através de cateteres diretamente no seio coronário ${ }^{5}$. Entretanto, a própria presença a válvula do seio coronário poderia servir como obstáculo para a introdução do cateter, dificultando a sua perfeita localização e conseqüente infusão da solução. Contribuem para isto o fato de que, na maioria das vezes, esta introdução é feita às cegas, apenas por palpação externa para posicionamento do cateter no interior do seio coronário. Além da cardioplegia, os estudos eletrofisiológicos e métodos de eletrofulguração também podem se utilizar da cateterização do seio coronário e se deparar com a presença da válvula de Thebesius.

Trabalho realizado no Instituto do Coração do Hospital das Clínicas da Faculdade de Medicina da Universidade de Săo Paulo. Săo Paulo, SP. Brasil. 
JATENE, F. B.; COSTA SOBRINHO, R. B.; ROMERO, S. R. T.; JATENE, A. D. - Estudo anatómico da válvula do seio coronário: válvula de Thebesius. Rev. Bras. Cir. Cardiovasc., 4(2): 163-167, 1989.

Este estudo foi realizado com o intuito de melhor conhecer as várias disposições da válvula de Thebesius, visando fornecer dados para melhor cateterização do seio coronário.

\section{CASUÍSTICA E MÉTODOS}

Foram estudadas as variaçōes anatômicas da válvula de Thebesius em 94 coraçōes de individuos falecidos de causa não cardiaca. Todos os coraçōes eram anatomicamente normais, sendo $80(85 \%)$ pacientes do sexo masculino, com idades entre 13 a 86 anos (30 anos), sendo $56(60 \%)$ indivíduos de côr branca e $38(40 \%)$ não brancos.

O átrio direito era incisado ao nível da veia cava superior em direção à veia cava inferior, percorrendo o sulco terminal, permitindo uma exposição da cavidade atrial pela afastamento da porção superior do átrio direito. Desta maneira, foi possível visibilizar o septo interarial e o "assoalho" do átrio direito com apresentação adequada do óstio do seio coronário e regiảo adjacente (Figura 1). Este local era analisado cuidadosamente, com a perfeita identificação do seio coronário e da sua válvula, identificando seu tipo, formato, consistência e implantação. Em todas as peças, cateteres usados para eletrofulguraçăo e para estudo eletrofisiológico (7F bipolar ou tripolar), e cateter com balonete na sua extremidade, com $4 \mathrm{~mm}$ de diâmetro, foram introduzidos no seio coronário para avaliar a dificuldade desta manobra.

A seguir, era feito um desenho esquemático para cada peça, das estruturas do septo interatrial e, especialmente, da regiāo em questāo, englobando também a veia cava inferior e a valva tricúspide. Reproduziram-se, fielmente, os detalhes anatômicos da válvula de Thebesius e, com base em suas variantes anatômicas, foi possível sua classificação nos seguintes grupos: válvula ausente, residual, parcial, trabeculada e dupla.

No primeiro grupo, que abrangeu a válvula ausente, o óstio do seio coronário estava exposto e não existia válvula propriamente dita, possibilitando, assim, uma comunicação direta com o átrio direito (Figura 2A).

No segundo grupo, a válvula foi designada como válvula residual. Nesses coraçōes, o óstio do seio coronário possuia uma pequena membrana, não superior a $15 \%$, o diâmetro do óstio do seio coronário, inserida em sua borda ínfero-direita, permitindo, também, uma ampla comunicação com a cavidade atrial (Figura 2B).

No terceiro grupo, o óstio possuía uma membrana recobrindo-o parcialmente (acima de $15 \%$ do seu diâmetro). Essa membrana, inserida a partir da borda inferior

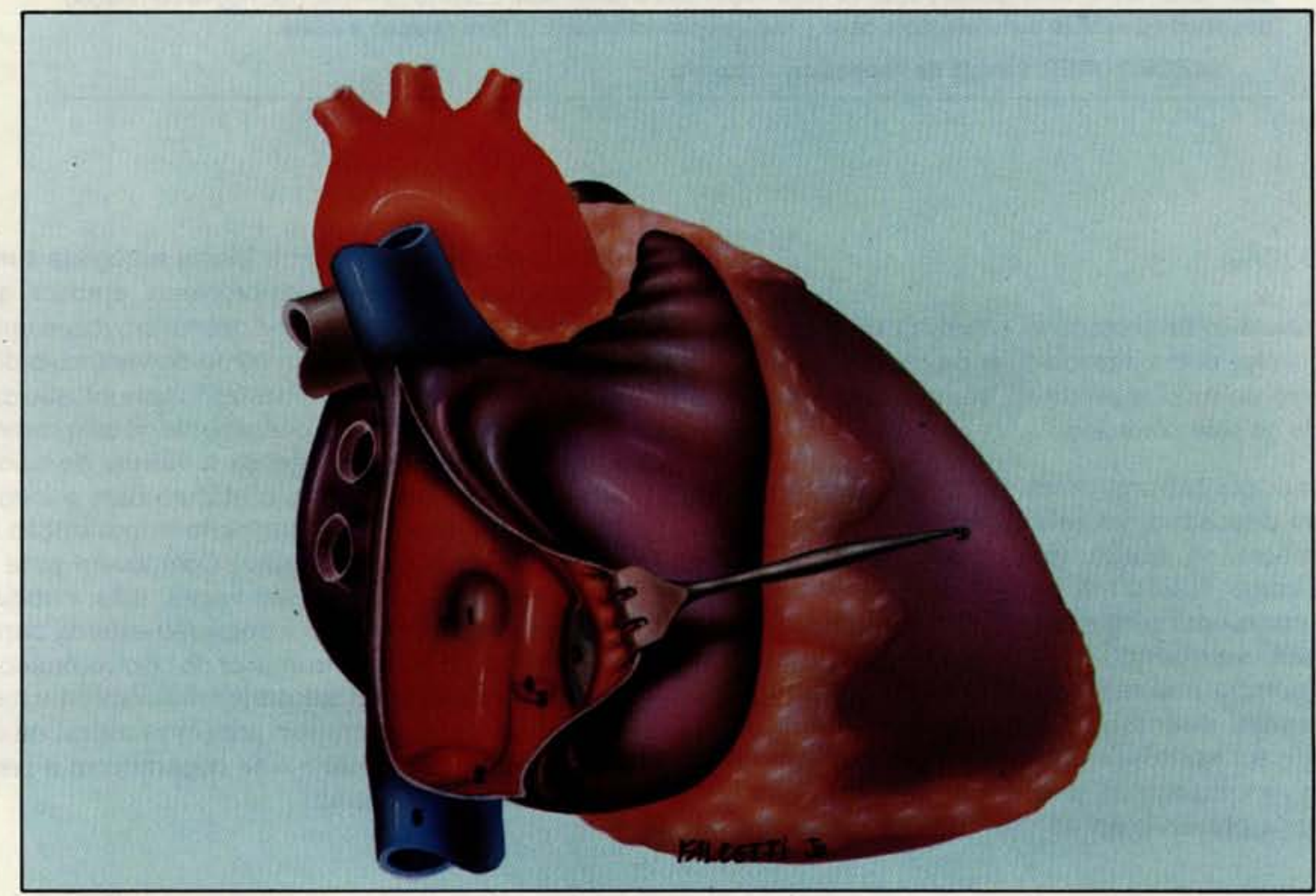

Fig. 1 - Esquema do átrio direito aberto mostrando as suas estruturas: 1) fossa oval;2) válvula de Eustáquio; 3) válvula de Thebesius: 4) orificio do seio coronário; 5) valva tricúspide; 6) veia cava inferior. 
JATENE, F. B.; COSTA SOBRINHO, R. B.; ROMERO, S. R. T.; JATENE, A. D. - Estudo anatômico da válvula do seio coronário: válvula de Thebesius. Rev. Bras. Cir. Cardiovasc., 4(2): 163-167, 1989.

e também nas bordas direita e esquerda do óstio, com extremidade livre e forma côncova, recebeu o nome de válvula parcial. Este tipo valvular foi subdividido, de acordo com suas características próprias, em parcial não perfurada quando a membrana se encontrava integra, parcial perfurada quando apresentava pequenos orifícios em sua membrana e parcial com traves quando a válvula apresentava uma ou mais traves partindo de sua membrana em sentido à borda do seio coronário (Figura 2C, D, E).

No quarto grupo, a válvula foi demonimada trabeculada quando o óstio do seio coronário não possuía membrana e sim traves atravessando toda sua extensão. Este grupo foi subdividido em simples quando as traves não apresentavam conexão entre si e reticulada quando se conectavam, formando uma fina rede sobre o óstio do seio coronário (Figura $2 \mathrm{~F}, \mathrm{G}$ ).

Um quinto grupo, abrangeu a válvula dupla. $O$ seio coronário, neste caso, possuía duas membranas, dispostas em planos distintos e cada uma abrangendo cerca de $70 \%$ da superfície do óstio, sendo uma localidade na borda inferior e outra na borda superior do óstio coronário (Figura 2H).

\section{RESULTADOS}

$\mathrm{Na}$ Tabela 1 está representado o número de peças para cada grupo e seus subgrupos.

Os dados relativos a sexo, raça e idade para cada grupo estão representados na Tabela 2.

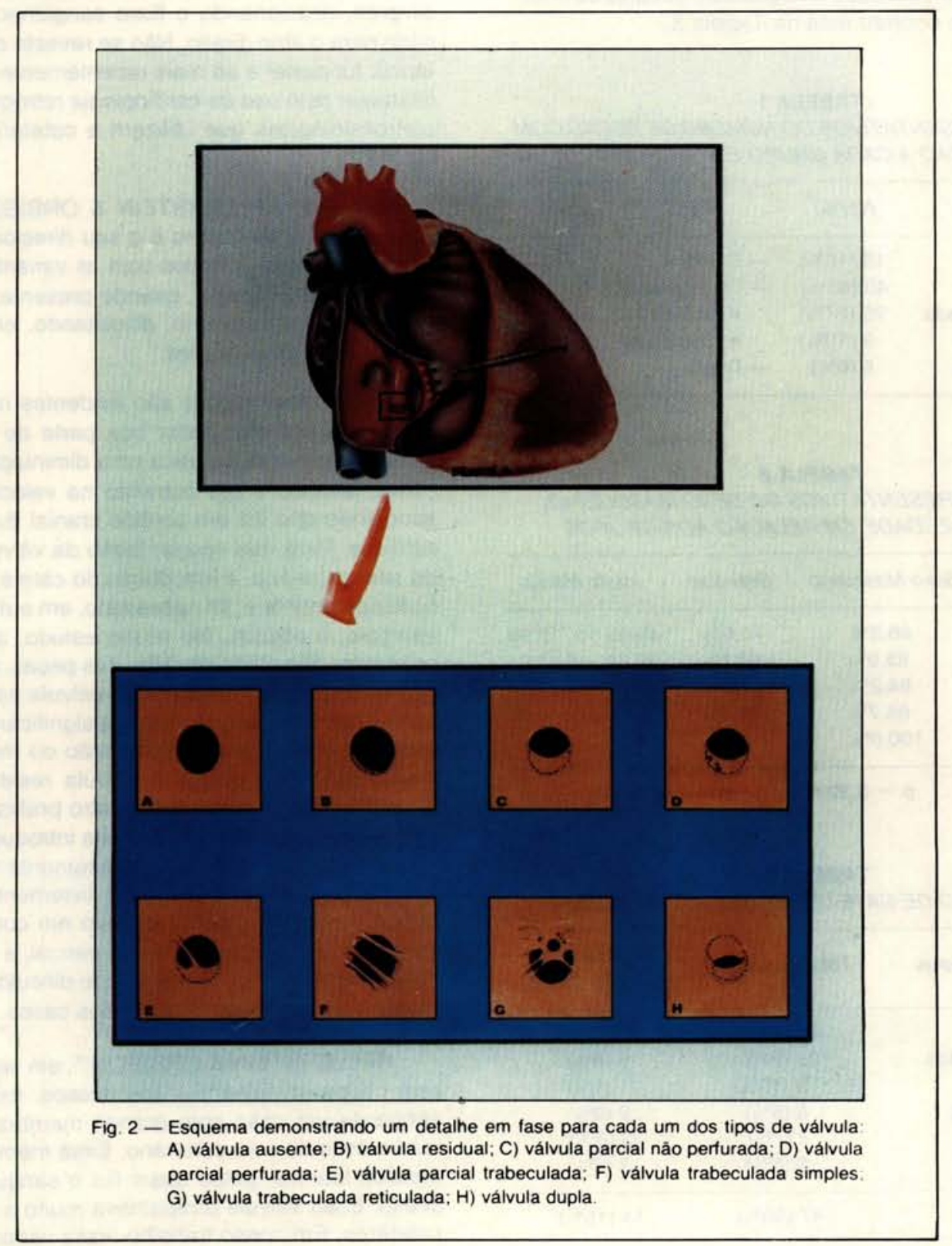


JATENE, F. B.; COSTA SOBRINHO, R. B.; ROMERO, S. R. T.; JATENE, A. D. - Estudo anatômico da válvula do seio coronário: válvula de Thebesius. Rev. Bras. Cir. Cardiovasc., 4(2): 163-167, 1989.

Sob o ponto de vista estatístico, não houve correlação significativa dos tipos de válvula com relação a sexo e raça. Embora seja significativa a ocorrência da válvula trabeculada em grupo mais jovem, em relação à válvula ausente no grupo mais idoso isto deve ser considerado com reserva devido à pequena amostra no grupo trabeculado.

A introdução de cateteres 7F bipolar ou tripolar $e$ cateter com balonete no interior do seio coronário foi obtida em todas as peças. Naqueles grupos onde a válvula era ausente, residual ou dupla, a introdução desses cateteres foi imediata. Entretanto, em algumas peças dos grupos onde ela era parcial ou trabeculada, a introdução do cateter-balão foi mais difícil e trabalhosa. Isto ocorreu em $14(15 \%)$ do total de peças avaliadas.

A descrição detalhada dos grupos e subgrupos onde esta dificuldade ocorreu está na Tabela 3.

TABELA 1

DADOS REPRESENTATIVOS DO NUMMERO DE PECAS COM RELAÇAO A CADA GRUPO E SUBGRUPO

\begin{tabular}{lccc}
\hline TIPO & N: $(\%)$ & TIPO & N. $(\%)$ \\
\hline - Ausente & $15(16 \%)$ & - Residual & $31(33 \%)$ \\
- Parcial & $40(43 \%)$ & - Trabeculada & $7(7 \%)$ \\
- não perfurada & $25(27 \%)$ & - simples & $5(5 \%)$ \\
- perfurada & $9(10 \%)$ & $\bullet$ reticulada & $2(2 \%)$ \\
- com traves & $6(6 \%)$ & - Dupla & $1(1 \%)$ \\
\hline
\end{tabular}

TABELA 2

DADOS REPRESENTATIVOS DO SEXO MASCULINO, BRANCOS E IDADE EM RELAÇATO AOS GRUPOS

\begin{tabular}{|c|c|c|c|}
\hline Grupos & Sexo Masculino & Brancos & Idade Média \\
\hline Ausente & $88,2 \%$ & $70,6 \%$ & $40,05 \pm 19,99$ \\
\hline Residual & $83.9 \%$ & $67,7 \%$ & $32,25 \pm 17,04$ \\
\hline Parcial & $84,2 \%$ & $50,0 \%$ & $28,51 \pm 11,02$ \\
\hline Trabeculada & $85,7 \%$ & $71,4 \%$ & $22,85 \pm 6,86$ \\
\hline Dupla & $100,0 \%$ & - & 48,0 \\
\hline
\end{tabular}

$p=0,979$ N.S. $p=0,32$ N.S. $p=0,0223$ Sig.

TABELA 3

INTRODUCÃO DE CATETER-BALÃO X DIFICULDADE

Grupos e Subgrupos Total peças (\%) Dificuldade de

\begin{tabular}{lcc} 
& & \\
\hline - Parcial & $40(43 \%)$ & $11(12 \%)$ \\
- Náo Perfurada & $25(27 \%)$ & $8(9 \%)$ \\
- Perfurada & $9(10 \%)$ & $1(1 \%)$ \\
- Com Traves & $6(6 \%)$ & $2(2 \%)$ \\
- Trabeculada & $7(7 \%)$ & $3(3 \%)$ \\
- Simples & $5(5 \%)$ & $3(3 \%)$ \\
\hline TOTAL & $47(50 \%)$ & $14(15 \%)$ \\
\hline
\end{tabular}

\section{DISCUSSĀO}

Em um número pequeno de casos, quando a válvula é trabeculada, a colocação do cateter é dificultada pela irregularidade com que as fibras estão dispostas sobre o orifício do seio coronário. Esta dificuldade só ocorreu, em nosso material, na presença da válvula trabeculada simples, ou seja, três em sete casos; quando introduzimos o cateter nas peças onde existia válvula trabeculada reticulada, não houve problemas, já que a membrana, nesse caso, se localizara na posição suspensa do óstio, de forma a possibilitar um diâmetro satisfatório para o manuseio do cateter. O fluxo sanüíneo, nesses coraçōes, nāo possui uma direçāo fixa como nos citados acima, fluindo em todas as direçōes na cavidade atrial.

A função da válvula de Thebesius é relativamente simples, direcionando o fluxo sangüíneo do seio coronário para 0 átrio direito. Não se reveste de maior importância funcional e só mais recentemente adquiriu maior destaque pelo uso da cardioplegia retrógrada e estudos eletrofisiológicos que utilizam a cateterização do seio coronário $^{2}$.

Segundo HELLERSTEIN \& ORBISON ${ }^{4}$, a velocidade do fluxo sangüíneo e o seu direcionamento estão intimamente relacionados com as variantes anatômicas da válvula, e a mesma, quando presente, diminui o diâmetro do seio coronário, dificultando, em raros casos, a introdução de cateteres.

Estas observaçōes são evidentes na presença da válvula parcial. Por cobrir boa parte de todo o orificio do seio coronário, provoca uma diminuição em seu diâmetro, levando a um aumento na velocidade do fluxo sangüíneo que flui em sentido cranial durante a sistole cardiaca. Para não ocorrer lesão da válvula, ou mesmo do seio coronário, a introdução do cateter deve ser feita cuidadosamente e, se necessário, em outra posição, por exemplo, a obliqua. No nosso estudo, a colocação do cateter foi dificultada em $15 \%$ das peças, principalmente nas que possuiam exuberante válvula parcial, e válvula parcial com traves, que diminuía significantemente o diâmetro disponivel para a colocaçāo do mesmo. Quando o seio coronário possui a válvula residual ou, então, a ausência da mesma, o diâmetro praticamente não se altera, possibilitando uma perfeita introdução do cateter. Como não há presença de praticamente nenhum obstáculo, o fluxo sangüíneo se faz livremente para o átrio direito e sua velocidade, quando em comparaçāo com coraçōes que possuem válvula parcial, é reduzida ${ }^{4}$. No nosso material, não houve grande dificuldade para introdução do cateter em nenhum dos casos.

HELLERSTEIN \& ORBISON ${ }^{4}$, em seu trabalho, trazem dados semelhantes aos nossos, exceto pela presença de um caso com grande membrana recobrindo todo o óstio do seio coronário. Essa membrana possuia orificios laterais, pelos quais flui o sangue para o átrio direito. Essa válvula atrapalhava muito a introdução de cateteres. Em nosso trabalho, essa variação anatômica 
JATENE, F. B.; COSTA SOBRINHO, R. B.; ROMERO, S. R. T.; JATENE, A. D. - Estudo anatômico da válvula do seio coronário: válvula de Thebesius. Rev. Bras. Cir. Cardiovasc., 4(2): 163-167, 1989.

não foi encontrada. Em contrapartida, tivemos um caso de dupla membrana, que, pela sua constituição anatômica, parecia dificultar a colocação do cateter. Entretanto, a cateterização desse seio coronário foi obtida de imediato, sem maiores dificuldades. HELLERSTEIN \& ORBISON ${ }^{4}$ também descrevem uma continuidade existente entre a válvula de Thebesius e a rede de Chiari. Esses casos são bem abordados por POWELL \& MULLANEY $^{6}$ e YATER ${ }^{8}$. São casos raros e não foram observados em nosso estudo. Contudo, em nosso material, algumas peças merecem atenção especial. Entre elas encontramos a válvula parcial, parcial perfurada, parcial trabeculada, e dupla membrana. Essas quadro válvulas possuem uma continuidade com a membrana de Eustáquio. Situações semelhantes a essa também são descritas na literatura por vários autores ${ }^{5}$, 6. 8. Além disso, em nosso estudo não houve nenhuma correlação estatística da válvula de Thebesius e seus respectivos tipos, quer seja com o sexo ou a raça. Embora esta correlação tivesse ocorrido com relação à idade (trabeculada nos mais jovens e tipo ausente nos mais idosos), isto não pode ser formalmente considerado em função do pequeno número de peças avaliado neste grupo.

Em análise final, a válvula de Thebesius não prejudica a introdução de cateter-balão, nas formas ausente, residual e dupla, mas casos especiais englobando algumas válvulas parciais e trabeculadas tornam este método um pouco mais trabalhoso e demorado; com relação ao estudo eletrofisiológico e à eletrofulguração, a válvula não dificulta o manuseio do cateter, de forma a possibilitar o adequado desenvolvimento deste estudo, não se constituíndo em um obstáculo para a cateterização do seio coronário.

Não há correlação com o tipo de válvula e o sexo ou raça, havendo indícios de correlação à idade.

RBCCV $44205-88$

JATENE, F. B.; COSTA SOBRINHO, R. B.; ROMERO, S. R. T.; JATENE, A. D. - Anatomical study of the coronary sinus valve: Thebesius valve. Rev. Bras. Cir. Cardiovasc., 4(2): 163-167, 1989.

ABSTRACT: The knowledge of the different anatomical types of the Thebesius valve should clarify some problems observed in coronary sinus catheterization. Ninety-four human normal hearts were analysed by an incision in he right atrium from the inferior vena cavae through the terminal groove, allowing inspection of the coronary sinus. Based on the anatomical features of the Thebesius valve, we identify 5 different groups, i.e.: 1) absent valve (16\%) when no valve exists; 2 ) residual valve $(33 \%)$ when there was only a small membrane occluding less than $15 \%$ of the sinus diameter; 3 ) partial valve $(43 \%)$ when a membrane occludes more than $15 \%$ of the sinus diameter; 4 ) trabeculated valve $(7 \%)$ when there was no membrane but trabeculation over the sinus; 5 ) double valve (1\%) when there were 2 membranes over the sinus in different positions. In all cases we tried to perform catheterization of the coronary sinus with 2 different types of catheters commonly used in hemodynamic studies. We observed more difficulty to catheterize the coronary sinus with partial or trabeculated valves. There was no statistical relationship between the different valves and age, sex or race.

DESCRIPTORS: Thebesius valve, anatomy.

\section{REFERÊNCIAS BIBLIOGRÁFICAS}

1 FABIANI, J.' N.; DELOCHI, A.; SWANSON, J.; CARPENTIER, A. - Retrograde cardioplegia throught right atrium. Ann. Thorac. Surg., 41: 101-102, 1986.

2 GALLAGHER, J. J.; PRITCHETT, E. L. C.; SEALLY, W. C.; KASELL, L.; WALLACE, A. G. - The pre-excitation syndromes. Progr. Cardiovasc. Dis., 20: 285-327, 1978.

3 GROSS, C. M. (ed.) - Gray's anatomy. 2.ed. Philadelphia, Lea and Febiger, 1977.

4 HELLERSTEIN, H. K. \& ORBISON, J. C. - Anatomic variation of the orifice of the human coronary sinus. Circulation, 3: 514-523, 1951.
5 MENASCHE, P.; KURAL, S.; FAUCHET, M.; LAVERGNE, A.; COMMIN, P.; BERCOT, M.; TOUCHOT, B.; GEORGIOPOULOS, G.; PIWNICA, M. - Retrograde coronary sinus perfusion: a safe alternative for insuring cardioplegic delivery in aortic valve surgery. $A n n$. Thorac. Surg., 34: 647-658, 1982.

6 POWELL, E. D. U. \& MULLANEY, J. M. - The Chiari network and the valve of the inferior vena cava. $B r$. Heart J., 22: 579-584, 1960.

7 TESTU, L. \& LATARJET, A. - Corazon y pericrdio. 9.ed. Salvat Editores S.A., 1951. p. 62-69.

8 YATER, W. M. - Variations and anomalous of the venous valves of the right atrium of the human heart. Arch. Pathol., 7: 418-441, 1929. 